\title{
ESTRATÉGIAS EDUCATIVAS EM SAÚDE NO CUIDADO ÀS PESSOAS COM DOENÇAS CRÔNICAS NÃO TRANSMISSÍVEIS
}

\author{
Luciele Janner Budel1; Cláudia Millani Gomes²; Ana Lúcia de Freitas \\ Saccol ${ }^{3} J u l i a n a$ Silveira Colomé 4
}

\begin{abstract}
RESUMO
Objetivo: discutir estratégias educativas em saúde no cuidado às pessoas com doenças crônicas não transmissíveis. Método: Trata-se de um relato de experiência apresentado à Disciplina Educação em Saúde do Curso de Mestrado em Ciências da Saúde e da Vida da Universidade Franciscana, durante o primeiro semestre de 2021. As experiências ocorreram no setor privado, mais especificamente no Gerenciamneto de Doenças Crônicas e, ainda, no Ambulatório Regional Especializado de Diabetes Mellitus e Hipertensão Arterial Sistêmica, do setor público. Ambos os serviços estão localizados na cidade de Santa Maria - RS. Resultados: as principais estratégias educativas estão relacionadas ao autocuidado apoiado, às abordagens grupais, ao tele monitoramento e ao envolvimento familiar, comunitário e da equipe interprofissional nas ações de cuidado. Também foram destacadas, de modo relacionado, a educação permanente das equipes e o fortalecimento das redes de atenção às DCNT, assim como o estabelecimento de linhas de cuidado e diretrizes clínicas. Conclusão: Considerando a complexidade envolvida nas DCNT, as estratégias aqui discutidas articulam as dimensões educativas e de cuidado em saúde, podendo podem ser implementadas e consideradas nos diversos pontos de atenção da rede, independente de serem ligados ao setor e público e/ou privado.
\end{abstract}

Palavras-chave: Educação em Saúde; Interprofissionalidade; Doenças Crônicas Não Transmissíveis.

Eixo Temático: Atenção Integral e Promoção à Saúde (AIPS).

\footnotetext{
${ }^{1}$ Enfermeira. Mestranda do Curso de Mestrado em Ciências da Saúde e da Vida da Universidade Franciscana - UFN. lucielejanner@gmail.com

${ }^{2}$ Psicóloga. Mestranda do Curso de Mestrado em Ciências da Saúde e da Vida da Universidade Franciscana - UFN. claudiamillanig@gmail.com

${ }^{3}$ Doutora em Nutrição. Professora do Curso de Mestrado em Ciências da Saúde e da Vida da Universidade Franciscana - UFN. alsaccol@ufn.edu.br

${ }^{4}$ Doutora em Enfermagem. Mestrado em Ciências da Saúde e da Vida da Universidade Franciscana - UFN. juliana@ufn.edu.br
} 


\section{INTRODUÇÃO}

As doenças crônicas não transmissíveis, conhecidas pela sigla DCNT, estão relacionadas a diversos fatores e tendem a apresentar um curso clínico que se altera no seu processo de evolução, podendo começar de forma gradual, com sintomas progressivos e fases de agudização que causam prejuízos na vida funcional do indivíduo, com prognóstico, em geral, incerto, tendo longa e indefinida duração. Estas têm tem sido consideradas responsáveis por cerca de $70 \%$ de todas as mortes no mundo, representando a causa de 38 milhões de mortes anuais. Desses óbitos, 16 milhões ocorrem, precocemente, em menores de menores de 70 anos de idade. Além disso, aproximadamente 28 milhões de óbitos ocorrem em países de baixa e média renda, (WHO, 2012).

As condições crônicas, especialmente as doenças crônicas, possuem um funcionamento diferentes das doenças agudas, elas tendem a iniciar de forma lenta e gradativa e se mantém por toda a vida do indivíduo, comumente elas tem vários, fatores determinantes de causalidade e um deles é a hereditariedade, estilos de vida, exposição a fatores ambientais e a fatores fisiológicos. Ao contrário do que acontece nos processos de adoecimento em doenças agudas, onde em geral, pode-se esperar uma recuperação adequada, as condições crônicas levam a mais sintomas e à perda de capacidade funcional (COSTA, 2016).

Estudos indicam que fatores como o tabaco, inatividade física, uso excessivo de álcool e dietas não saudáveis causam o aumento das DCNT. Desse modo, com a mudança nesses fatores, poderia haver, consequentemente, a redução do número de mortes em todo o mundo (WHO, 2012). O grande aumento das DCNTs, resultam em consequências devastadoras para a comunidade, os indivíduos e as famílias, além de sobrecarregar os sistemas de saúde a, (WHO, 2011). Estudos apontam que as populações de baixa renda, são as mais afetadas pelas DCNTs, por estarem mais vulneráveis, mais expostas aos riscos e terem menor acesso aos serviços de saúde e às práticas de promoção à saúde e prevenção das doenças (WHO 2012). A Organização Mundial da Saúde (OMS) avalia que as pessoas com DCNT têm sua situação de pobreza agravada, pelos maiores gastos familiares com a doença pela procura de servicos, dentre outros (WHO , 2011). 
Além dessa quetsão, a economia acaba sofrendo significativamente 0 peso do alto número de portadores de doenças crônicas, assim ocontrole e redução das DCNT, se torna uma condição necessária para os países em geral (KELE, 2010). Nesse sentido, as pessoas acometidas de uma doença crônica, necessitam de um cuidado contínuo, como também mudanças no seu estilo de vida. O cuidado à pessoa com DCNT de ser desenvolvido de modo integral e necessita de condutas singularizem cada indivíduo, sem desconsiderar seu contexto ampliado de vida. Destaca-se que, quando o cuidado é generalizado, tende a não surtir os efeitos desejados, pois esse olhar não se torna fiel à demanda de cada um em particular (MALTA; MERHY, 2010).

Em função disso, o Sistema Unico de Saúde (SUS), no ano de 2014, redefiniu a Rede de Atenção à Saúde das Pessoas com Doenças Crônicas, estabelendo diretrizes para a organização das suas linhas de cuidado (MENDES, 2011). Nessa direção, as operadoras de planos de saúde, atentas às orientações da Agência Nacional de Saúde Suplementar (ANS), também estão implementando programas de prevenção e promoção da saúde, como o disposto na Resolução Normativa 264/2011, que preconiza o desenvolvimento de ações de Promoção da Saúde e Prevenção de Riscos e Doenças em seus programas de saúde suplementar (BRASIL, 2013).

Considerando o exposto, esse trabalho tem como objetivo discutir estratégias educativas em saúde no cuidado às pessoas com doenças crônicas não transmissíveis.

\section{2- METODOLOGIA}

Trata-se de um relato de experiência apresentado à Disciplina Educação em Saúde do Curso de Mestrado em Ciências da Saúde e da Vida da Universidade Franciscana, durante o primeiro semestre de 2021. Em função da atuação pregressa das mestrandas em cenários de cuidado às DCNT, houve a escolha da temática relacionada às estratégias educativas em saúde nesse contexto. As reflexões expressas nesse trabalho foram apresentadas em um Seminário Temático na disciplina.

As experiências das autoras principais ocorreram no período entre os anos de 2012 a 2018 no setor privado, mais especificamente no Gerenciamneto 
de Doenças Crônicas e, entre os anos de 2018 e 2019, no Ambulatório Regional Especializado de Diabetes Mellitus e Hipertensão Arterial Sistêmica, do setor público. Ambos os serviços estão localizados na cidade de Santa Maria - RS.

\section{RESULTADOS E DISCUSSÕES}

As experiências vivenciadas no contexto do cuidado aos usuários com DCNT envolveram, em ambos os cenários, estratégias que envolvem práticas educativas em saúde. Desse modo, essas são refletivas a seguir em sua articulação com a literatura. As estratégias educativas em saúde no contexto das DCNT requerem, necessariamente, o trabalho em equipe interprofissional. Essa atuação tem sido definida e se tornado importante como aquela que envolve diferentes profissionais, não apenas da saúde, que juntos compartilham o senso de pertencimento à equipe e trabalham juntos de maneira integrada e interdependente para atender às necessidades de saúde (SILVA, 2015).

Uma das possibilidades encontradas no fazer educativo interprofisisonal diz repeito ao envolvimento ativo dos usuários no seu processo de cuidado. Nas doenças crônicas, o sucesso do tratamento depende fortemente da participação e do envolvimento do usuário e, consequentemente, no seu autocuidado. Desse modo, mudanças nos estilos e práticas de vida mais saudáveis, assim como a adesão ao tratamento, não dependem apenas de uma prescrição profissional, mas de uma conscientização do usuário e familiares sobre sua condição de saúde e a relação dela com suas práticas (AGRELI; PEDUZI; SILVA 2016).

O autocuidado apoiado significa uma colaboração estreita entre a equipe de saúde e os usuários, os quais trabalham em conjunto para definir o problema, estabelecer as metas, monitorá-las, instituir os planos de cuidado e intervir nos problemas que apareçam ao longo do processo de manejo, melhorando seu prognóstico (AGRELI; PEDUZI; SILVA 2016). Os profissionais da saúde devem estar preparados para que colaborem com os usuários no estabelecimento de metas para o autocuidado, já que implica mudança de prática. Ainda, deve-se utilizar instrumentos de autocuidado baseados em evidências clínicas, explorando as estratégias grupais por meio de ações educativas, além de 
buscar recursos da comunidade para que as metas de autogerenciamento sejam obtidas (HEALTH DISPARITIES COLLABORATIVES, 2004 apud MENDES, 2011).

Nessa perspectiva, os grupos são dispositivos potentes de educação em saúde, trocas entre os usuários e destes com a equipe de saúde. Essa abordagem deve estimular a reflexão sobre o adoecimento e os fatores envolvidos nesse processo para, a partir disso, estimular formas de autocuidado e mudança de atitudes (MENDES, 2011). Os grupos podem ser organizados de diversas formas, adequadas ao objetivo proposto e ao perfil dos usuários. Devem ser privilegiadas abordagens criativas que envolvam a participação coletiva. A organização dos grupos possibilita integração e discussões, favorecendo a criação de redes de cuidado para além do grupo. Isso gera sujeitos ativos e espaço onde as pessoas podem superar suas dificuldades e obter maior autonomia e, também, estreitar a relação entre a equipe interprofissional e o usuário (ALMEIDA; SOARES, 2010; BRASIL, 2010).

O apoio da família, dos amigos, das organizações comunitárias e da equipe interprofissional é essencial para que o autocuidado se dê com efetividade (COSTA, 2016). As recomendações específicas para cada usuário devem ser elaboradas com o objetivo de orientar o cuidado, a partir da compreensão ampliada do processo saúde-doença, com foco na integralidade. As orientações das equipes não substituem a interação, o diálogo e a escuta. Para tanto, a educação permanente deve ser valorizada, tendo em vista que a atuação nas DCNT implica mudanças nos processos de trabalho, sendo fundamental que a educação permanente das equipes supere os modelos tradicionais de educação e caminhe no sentido de estratégias que valorizem o trabalhador, seu conhecimento prévio e sua experiência profissional (PEDUZZI, 2001).

Outra possibilidade envolvida no cuidado das DCNT tem sido utilizada no setor público de saúde e, ainda, por algumas operadoras de saúde, que implementaram setores de medicina preventiva a partir do serviço de tele monitoramento voltado para o acompanhamento de usuários com doenças crônicas. Esse modelo pode ter diversos formatos, ativos ou receptivos, de acordo com as prioridades estabelecidas pelo sistema de saúde. Especialmente 
no contexto da pandemia por Covid-19, essa estratégia apresenta-se como pertinente e necessária nos serviços de saúde.

As ligaçoes telefônicas servem como fortes aliadas aos programas de educaçao em saúde e nos planos de autocuidado e Projetos Terapêuticos Singulares, também sendo utilizadas no pós-alta hospitalar. Nos casos de maior complexidade, essa ação também é eficaz, pois torna a necessidade de consultas menos necessárias (SILVA, 2015). No acompanhamento não presencial, as interações entre as equipes de saúde e os usuários podem se tornar mais produtivas com a articulação entre atendimentos profissionais presenciais e não presenciais, por meio de telefone ou de correio eletrônico (MENDES, 2011).

No contexto da Atenção Básica em Saúde, as DCNT se mostram como uma grande demanda no cotidiano das unidades de saúde, grupos e visitas domiciliares. Estas condições são muito prevalentes, multifatoriais com coexistência de determinantes biológicos e socioculturais, e sua abordagem, para ser efetiva, necessariamente envolve as diversas categorias profissionais das equipes de Saúde e exige o protagonismo dos indivíduos, suas famílias e comunidade. (AGRELI; PEDUZI; SILVA 2016).

Nesse contexto, o Ministério da Saúde vem desenvolvendo diretrizes, metodologias e instrumentos de apoio às equipes de Saúde e realizando um esforço para que se organize a Rede de Atenção às Pessoas com Doenças Crônicas. Esta rede visa qualificar o cuidado integral, unindo e ampliando as estratégias de promoção da saúde, de prevenção do desenvolvimento das doenças crônicas e suas complicações, e de tratamento e recuperação (MENDES, 2012).

Organizar as linhas de cuidado e diretrizes clínicas expressam os fluxos assistenciais que devem ser garantidos ao usuário no sentido de atender às suas necessidades de saúde. As linhas definem as ações e os serviços que devem ser desenvolvidos nos diferentes setores de atenção a saúde Essas normatizam todo o processo da condição de saúde ao longo da sua história natural, incluindo ações promocionais, preventivas, curativas, cuidadoras, reabilitadoras e paliativas relativas à determinada doença (FRANCO; FRANCO, 2012; MENDES, 2011). 
Percebe-se ainda a necessidadede de uma mudança de paradigma do sistema de saúde, pois deve-se ainda trabalhar na construção de redes que vem ao encontro deste grande desafio, visto que o trabalho da saúde em redes, vem propondo o fortalecimento de vínculos entre a população e os pontos de atenção, o que é fundamental para a atenção à saúde integral da pessoa com doenças crônicas, independente do modelo institucional. Essa construção requer os esforços de todos os atores envolvidos, principalmente quando se trata da articulação e da comunicação entre os componentes dos modelos de atuação.

\section{CONCLUSÃo}

As estratégias educativas em saúde nas DCNT podem ser potencializadas pela atuação de equipe interprofissional que envolva o usuário nos processos de autocuidado. Tendo em vista a necessidade de atenção singular para o autocuidado apoiado, há que se garantir espaços de análise e discussão de casos nas equipes, com a possibilidade de analisar e discutir cada caso, a fim de articular os saberes de diversos núcleos profissionais. Considerando a complexidade envolvida nas DCNT, as estratégias aqui discutidas articulam as dimensões educativas e de cuidado em saúde, podendo podem ser implementadas e consideradas nos diversos pontos de atenção da rede, independente de serem ligados ao setor e público e/ou privado.

\section{REFERÊNCIAS}

AGRELI HF, PEDUZI M, SILVA MC. Atenção centrada no paciente na prática interprofissional colaborativa. Interface (Botucatu). 2016; 20(59):905-16.

ALMEIDA, S. P.; SOARES, S. M. Aprendizagem em grupo operativo de diabetes: uma abordagem etnográfica. Ciênc. Saúde Coletiva, [S.I.], São Paulo, v. 15, p. 1123-1132, 2010.

BRASIL. Decreto № 7.508, de 28 de junho de 2011. Regulamenta a Lei no 8.080, de 19 de setembro de 1990. Dispõe sobre a organização do Sistema Único de Saúde (SUS), o planejamento da saúde, a assistência à saúde e a articulação 
interfederativa, e dá outras providências. Diário Oficial da União, Poder Executivo, Brasília, DF, 29 jun. 2011.

COSTA MV. A educação interprofissional no contexto brasileiro: algumas reflexões. Interface (Botucatu). 2016; 20(56):197-8.

FRANCO, C. M.; FRANCO, T. B. Linhas do cuidado integral: uma proposta de organização da rede de saúde, 2012.

MALTA, D. C.; MERHY, E. E. O percurso da linha do cuidado sob a perspectiva das doenças crônicas não transmissíveis. Interface: comunic., saúde, educ., Botucatu, SP, v. 14, n. 34, p. 593-605, jul./set. 2010.

MENDES, E. V. As redes de atenção à saúde. Brasília: Organização PanAmericana da Saúde, 2011.

MINISTÉRIO DA SAÚDE, Agência nacional de saúde suplementar. Agência reguladora do setor de planos de saúde do Brasil. https://www.gov.br/ans/pt-br MINISTÉRIO DA SAÚDE, Secretaria de Atenção à Saúde Departamento de Atenção Básica Diretrizes para o cuidado das pessoas com doenças crônicas nas redes de atenção à saúde e nas linhas de cuidado prioritárias. Brasília - DF 2013.

OMS. Organização Mundial da Saúde. Framework for action on interprofessional education and collaborative practice [Internet]. Geneva: OMS; 2010 [citado 4 Abr2017].Disponível em: http://www.who.int/hrh/resources/framework action/en/

SILVA J. A. et al. Educação interprofissional e prática colaborativa na Atenção Primária à Saúde. Rev Esc Enferm USP. 2015;

WORLD HEALTH ORGANIZATION. Global status report on noncommunicable diseases 2010. Geneva: World Health Organization; 2011. 
WORLD HEALTH ORGANIZATION. Health statistics and information systems: estimates for 2000-2012. Geneva, 2012. 